\title{
Single-cell Analysis of Lambda Immunity Regulation
}

Bæk, Kristoffer Torbjørn; Svenningsen, Sine Lo; Eisen, Harvey; Sneppen, Kim; Brown, Stanley

Published in:

Journal of Molecular Biology

DOI:

10.1016/j.jmb.2003.09.037

Publication date:

2003

Document version

Early version, also known as pre-print

Citation for published version (APA):

Bæk, K. T., Svenningsen, S. L., Eisen, H., Sneppen, K., \& Brown, S. (2003). Single-cell Analysis of Lambda Immunity Regulation. Journal of Molecular Biology, 334(3), 363-372. https://doi.org/10.1016/j.jmb.2003.09.037 


Available online at www.sciencedirect.com

\section{Single-cell Analysis of $\boldsymbol{\lambda}$ Immunity Regulation}

\section{Kristoffer Bæk1, Sine Svenningsen', Harvey Eisen², Kim Sneppen ${ }^{3}$ and Stanley Brown ${ }^{1 *}$}

\author{
${ }^{1}$ Department of Molecular \\ Cell Biology, University of \\ Copenhagen, Øster \\ Farimagsgade 2A, DK-1353 \\ Copenhagen K, Denmark \\ ${ }^{2}$ Fred Hutchinson Cancer \\ Research Center \\ 1100 Fairview Avenue North \\ Seattle, WA 98109, USA \\ ${ }^{3}$ Niels Bohr Institute \\ Blegdamsvej 17, DK-2100 \\ Copenhagen $\oslash$, Denmark
}

\begin{abstract}
We have examined expression of the $\lambda c I$ operon in single cells via a rex:: $g f p$ substitution. Although average fluorescence agreed with expectations for expression of $\lambda$-repressor, fluorescence fluctuated greatly from cell-tocell. Fluctuations in repressor concentration are not predicted by previous models and are tolerated in part by a regulatory response to DNA damage.
\end{abstract}

(C) 2003 Elsevier Ltd. All rights reserved.

\section{Introduction}

When phage $\lambda$ infects a sensitive Escherichia coli, it responds to a variety of intracellular signals and enters one of two developmental pathways. If it enters the lytic pathway, after about an hour the bacterium lyses releasing on the order of 100 progeny phage. If it enters the lysogenic pathway, most phage genes become repressed and the phage genome is integrated into the bacterial chromosome where it is passively replicated. The decision-making process by $\lambda$ was the first epigenetic switch to be deciphered and has been the subject of extensive study. ${ }^{1}$

The lysogenic state is maintained by the continued synthesis of a prophage-encoded repressor, the product of the $\lambda c I$ gene. Repressor binds to two operators, $O L$ and $O R$ and thereby turns off transcription from the lytic promoters $p L$ and $p R$, respectively. Binding of repressor to $o R$ also stimulates the promoter directing repressor synthesis in a lysogen, $p R M .^{1}$ Departure from the lysogenic state and entry into lytic development generally occurs only through the SOS response, the E. coli system for responding to DNA damage. ${ }^{2}$ In the

Abbreviations used: GFP, gene fusion protein; UV, ultraviolet light; IPTG, isopropyl thio- $\beta$-D-galactoside; m.o.i., multiplicity of infection; PFU and CFU, plaque and colony-forming units, respectively.

E-mail address of the corresponding author: stanley@biobase.dk absence of a functional SOS system (recA strains), departure from the lysogenic state is rare, occurring at a frequency of less than once per million cell-generations. ${ }^{3}$ In fact, the failure of lysogenic maintenance is less frequent than mutational inactivation of the $c I$ gene. ${ }^{4}$

The regulation of repressor synthesis has also been the subject of extensive study. ${ }^{1}$ Much of our understanding of the regulation of $c I$ expression derives from gene fusion studies. ${ }^{5-7}$ The gene fusion studies uncovered the basic features of $c I$ regulation and led to a molecular model for the epigenetic switch controlling induction of the $\lambda$ prophage. 8 In this model, repressor concentration in the lysogen must be maintained at precise levels to permit both stability of the prophage and departure from lysogeny in response to DNA damage. Also according to the model, the majority of regulatory events maintaining proper repressor concentration are mediated by repressor acting at $o R$, which is a constellation of three operators, $o R 1, o R 2$ and $o R 3$ (Figure. 1). These operators overlap two divergent promoters, the lytic promoter, $p R$, and the promoter directing synthesis of repressor in the lysogen, $p R M$. The $p R M-o R-p R$ region is compact. The distance separating the start sites of the two transcripts is 82 base-pairs. Binding of repressor to $o R$ is asymmetric; that is, repressor binds preferentially to $o R 1$, which overlaps $p R$, and only at higher concentrations to $o R 3$, which overlaps $p R M$. Binding of repressor to $o R 1$ blocks expression of $p R$ and cooperative binding to $o R 2$ 

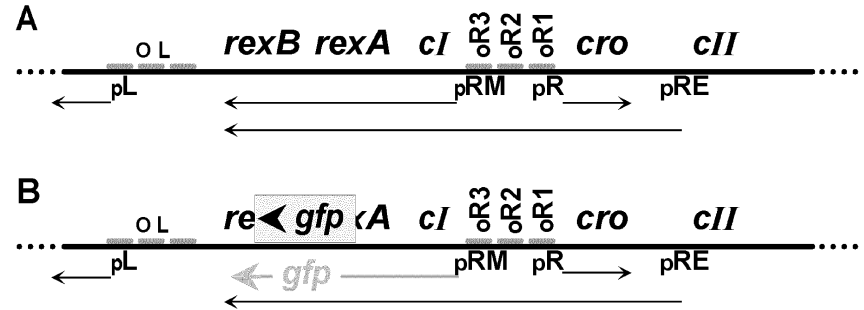

Figure 1. Immunity region of phage $\lambda$. Promoters for $c I$ transcription are $p R E$, the promoter for repressor establishment, and $p R M$, the promoter for repressor maintenance. The three operators of $o L$ and $o R$ are shown as are the lytic promoters $p L$ and $p R$. rexA and rexB are co-transcribed with $c I$. B, The substitution replacing parts of rexA and rexB with $g f p$. Features in this Figure are not drawn to scale. stimulates expression of $c I$ from $p R M$. Thus, the asymmetry of $o R$ readily explains the synthesis of repressor and the repression of lytic functions in a lysogen.

Since the early measurements of $c I$ expression were made on gene fusions separated from the other elements of the immunity region such as $o L$, features intrinsic to the context of the immunity region were not investigated. Recent reports suggest more features regulate the immunity of phage $\lambda$ than previously proposed. First is the participation of $o L$ in repression at $o R, 9,10$ consistent with the crystal structure of repressor. ${ }^{11} \mathrm{oL}$ overlaps $p L$, the lytic promoter for the left operon of $\lambda$ (Figure 1). A second and more surprising observation is the lack of a requirement for asymmetry at $o R \cdot{ }^{12}$ Models relying on asymmetry are difficult to reconcile with the observations by Little and co-workers ${ }^{12}$ that phage with a symmetric $o R$ form stable lysogens that can induce and grow lytically. The ability to form stable lysogens for mutants with symmetric operators suggests three possible scenarios for control of $p R M$. First, although all or nearly all transcription of $c I$ in a lysogen is initiated at $p R M,{ }^{13}$ there may be a second promoter responsible for a small amount of $c I$ in a lysogen. Second, $p R M$ may be substantially repressed in a wildtype lysogen and thus regulation of $p R M$ tolerates or perhaps relies on repression. The second model is especially attractive in light of the observation that $p R M$ is at least $60 \%$ repressed in a lysogen. ${ }^{10}$ A third model would rely on a higher-order complex including $o L$ to generate the asymmetry at $o R$, reducing the requirement for asymmetry at $o R$ itself.

\section{Results and Discussion}

\section{Role of $p R E$ in stability of lysogens}

We tested the contribution of another promoter to synthesis of repressor in a lysogen. The most likely candidate for a second promoter expressing $c I$ is $p R E$, the promoter active in the establishment of repression. Reichardt \& Kaiser ${ }^{14}$ found the concentration of repressor to be slightly lower from prophages defective in $p R E$-initiated transcription. Although they concluded the decrease was not sig- nificant, the observations cited above suggested the role of $p R E$ in maintaining lysogeny should be re-examined. If $p R E$ contributed to repressor synthesis in lysogens, prophage mutated in $p R E$ (cy) or its activator, CII, would display reduced stability of the lysogenic state. A decrease in lysogenic stability would result in a higher frequency of spontaneously released phage. We prepared single lysogens of $\lambda^{+}, \lambda c y$ and $\lambda c I I$ in a $\operatorname{rec} A$ bacterium and measured the frequency of released phage (Materials and Methods). The results of our measurements are described in Table 1. It can be seen in Table 1A that the frequency of total released phage was not elevated in lysogens defective in activating $p R E$. This observation suggests $p R E$ is unlikely to contribute to repressor synthesis in lysogens.

The $\lambda^{+}$lysogens release phage able to form both clear and turbid plaques. The majority of phage released are $c I$ mutants and form clear plaques. Presumably, the phage forming turbid plaques are wild-type and result from the spontaneous failure of the regulatory circuit maintaining lysogeny. $\lambda c y$ phage form clear plaques independently of whether they contain a wild-type or mutant $c I$ allele. To distinguish the two possible types of phage released from the $\lambda c y$ lysogens, we tested the ability of the released phage to provide repressor in the lysogenic state. We tested their ability to complement a lysogen of $\lambda c I^{t s}(\lambda c 1857)$ permitting bacterial growth at $42{ }^{\circ} \mathrm{C}$. It can be seen in Table $1 B$ that of 40 phage released from $\lambda c y$ lysogens, none was able to complement $\lambda c I^{t s}$ as efficiently as the $\lambda c y$ parents. Hence, these 40 phage were released due to mutations rather than due to failures of lysogenic regulation. This observation supports the above conclusion that $p R E$ is unlikely to direct sufficient repressor synthesis to make a substantial contribution to the stability of established lysogens. If $p R M$ is the sole source of repressor in lysogens, the ability of the symmetric oR mutants to form stable lysogens must be due to the ability of $p R M$ to tolerate strong repression or the ability of $o L$ to generate asymmetry at $o R$.

The $c I$ complementation behavior of phage released from the $\lambda^{+}$lysogen was examined as a control for the $c y$ experiment above. We expected the clear plaque-forming phage to complement $c I^{t s}$ poorly and it can be seen in Table 1 that of ten 
Table 1. Measurement of phage release and ability to complement a temperature-sensitive repressor

\begin{tabular}{|c|c|c|c|c|c|}
\hline \multicolumn{5}{|c|}{ A. Frequency of released phage from $p R E$ lysogens } & \\
\hline$\underline{\text { Strain }}$ & Prophage & Turbid & Clear & \multicolumn{2}{|l|}{ Total } \\
\hline KB12 & $\lambda w t$ & $\begin{array}{c}11 \\
3.9 \\
2.2 \\
3.1\end{array}$ & $\begin{array}{l}14 \\
6.9 \\
8.1 \\
8.1\end{array}$ & $\begin{array}{c}25 \\
10.8 \\
10.3 \\
11.2\end{array}$ & \\
\hline KB13 & $\lambda c y 42$ & & $\begin{array}{l}25 \\
16 \\
13 \\
6.5\end{array}$ & $\begin{array}{l}25 \\
16 \\
13 \\
6.5\end{array}$ & \\
\hline KB14 & $\lambda c y 2001$ & & $\begin{array}{l}24 \\
22 \\
13 \\
17\end{array}$ & $\begin{array}{l}24 \\
22 \\
13 \\
17\end{array}$ & \\
\hline KB15 & $\lambda c I I 68$ & & 6.6 & 6.6 & \\
\hline KB16 & $\lambda c I I 2002$ & & $\begin{array}{l}9.6 \\
15\end{array}$ & $\begin{array}{l}9.6 \\
15\end{array}$ & \\
\hline \multicolumn{3}{|c|}{ B. Ability of released phage to complement a cI857 lysogen for growth at $42^{\circ} \mathrm{C}$} & \multicolumn{3}{|c|}{$\begin{array}{l}\text { Efficiency of transduction to } 42{ }^{\circ} \mathrm{C}^{\mathrm{R}} \\
\left(10^{-6} \mathrm{CFU} / \mathrm{PFU}\right)\end{array}$} \\
\hline Lysate & Plaques analyzed & Experiment & Avg. & Min. & Max. \\
\hline$\lambda w t$ phage & & & $\begin{array}{l}1445 \\
2290 \\
2483\end{array}$ & & \\
\hline Turbids released from KB12 & 10 & $\begin{array}{l}\text { cult. A } \\
\text { cult. B }\end{array}$ & 21 & $\begin{array}{l}7.5 \\
2.4\end{array}$ & $\begin{array}{l}36 \\
44\end{array}$ \\
\hline Clears released from KB12 & 5 & $\begin{array}{l}\text { cult. A } \\
\text { cult. B }\end{array}$ & $\begin{array}{l}9.2 \\
6.0\end{array}$ & $\begin{array}{l}3.5 \\
1.9\end{array}$ & $\begin{array}{l}21.2 \\
11.8\end{array}$ \\
\hline$\lambda c y 42$ phage & & & $\begin{array}{l}2100 \\
1363\end{array}$ & & \\
\hline Clears released from KB13 & 10 & $\begin{array}{l}\text { cult. A } \\
\text { cult. B }\end{array}$ & $\begin{array}{l}6.0 \\
6.0\end{array}$ & $\begin{array}{l}0.64 \\
0.42\end{array}$ & $\begin{array}{l}12.7 \\
17.2\end{array}$ \\
\hline $\begin{array}{l}\lambda c y 2001 \text { phage } \\
\text { Clears released from KB14 }\end{array}$ & 10 & $\begin{array}{l}\text { cult. A } \\
\text { cult. B }\end{array}$ & $\begin{array}{l}2210 \\
7.5 \\
5.0\end{array}$ & $\begin{array}{l}0.7 \\
1.5\end{array}$ & $\begin{array}{c}15.6 \\
11\end{array}$ \\
\hline
\end{tabular}

Measurement of phage release and ability to complement a temperature-sensitive repressor are described in Materials and Methods.

phage examined, all complemented at least 100fold less efficiently than the $\lambda^{+}$parent. Surprisingly, all 20 of the turbid plaque-forming phage examined, although able to form turbid plaques at both $37^{\circ} \mathrm{C}$ and $42^{\circ} \mathrm{C}$, were inefficient in complementing $c I^{t s}$. That is, the released phage which form turbid plaques are not wild-type but are mutated in their ability to produce repressor in lysogens. Our results are consistent with findings of R. A. Roberts \& J. W. Little (University of Arizona) that, among the turbid-plaque-forming phage released from a $\operatorname{rec} A$ lysogen, the vast majority are not wild-type but carry a down-promoter mutation in $p R M$ which destabilizes the lysogenic state (personal communication). Since the released phage able to form turbid plaques are not wild-type, their release is not due to failure of the lysogenic regulatory circuit. Therefore, the lysogenic regulatory circuit must be much more stable than previously believed.

\section{Gene fusion to measure transcription of cl in situ}

To examine $c I$ transcription in single cells, we prepared a gene fusion to $g f p$. Since the presence of $o L$ increases repression at $o R,{ }^{9,10}$ we wanted to retain the surrounding prophage DNA in case other structures participated in regulation of $c I$ transcription. To this end, we prepared a gene fusion that maintained the wild-type spacing and surrounding DNA at the immunity region. To retain these features we substituted most of rexA and $r e x B$, two genes of the $c I$ operon, with the mut2 allele of $g f p$ rendering $g f p$ cotranscribed with $c I$ (Figure 1B; Materials and Methods). In the rex:: $g f p$ phage, $o L$ and $o R$ are separated by $2.2 \mathrm{~kb}$ compared with a wild-type spacing of $2.3 \mathrm{~kb}$.

GFPmut2 is stable ${ }^{15}$ and matures rapidly. ${ }^{16,17}$ Once protein synthesis was blocked (Materials and Methods), we observed no change in 
Table 2. Plasmids and hosts used in this study

\begin{tabular}{|c|c|c|c|c|}
\hline \multirow[b]{2}{*}{ Plasmid } & \multicolumn{4}{|c|}{ Average fluorescence } \\
\hline & $\begin{array}{c}\mathrm{p} c I 60 \\
c I^{-o R-}-\end{array}$ & $\begin{array}{c}\mathrm{p} c I^{+} \\
c I^{+o R}-\end{array}$ & $\begin{array}{l}\text { pUC18 } \\
\text { vector }\end{array}$ & $\begin{array}{c}\text { pSS5 } \\
C I^{+o R+}\end{array}$ \\
\hline \multicolumn{5}{|l|}{ Host } \\
\hline KB124 $\left(\right.$ Arex $\left.^{+}\right)$ & 1.2 & 2.2 & 1.4 & $<1$ \\
\hline KB126 $(\lambda r e x:: g f p)$ & 13.3 & 2.4 & 15.6 & $2.5(15.7)$ \\
\hline SS122 (גrex $:: g f p \Delta o L)$ & 32.8 & 1.2 & 39.8 & $3.7(46.4)$ \\
\hline \multicolumn{5}{|c|}{$\begin{array}{l}\text { Average fluorescence for each strain is described. rex:: } g f p \\
\text { lysogens harboring pSS5 showed a bimodal distribution of } \\
\text { fluorescence. The average fluorescence of the minority popu- } \\
\text { lation is shown in parentheses. The highly fluorescing popu- } \\
\text { lation varied from } 10 \% \text { to } 40 \% \text { in various experiments. }\end{array}$} \\
\hline
\end{tabular}

fluorescence during the course of our measurements. Therefore, fluorescence represents GFP concentrations in the cells upon withdrawal from the growing culture.

\section{Regulation of rex:: gfp}

In order to test whether $g f p$ expression in individual cells reflected repressor concentrations, we first verified that $g f p$ showed the regulation expected for $c I$. We supplied excess repressor from a multicopy plasmid expressing repressor from the lac promoter, $\mathrm{pcI}^{+}$. Both $\mathrm{pcI}^{+}$and the control plasmid bearing the defective cI60 allele, pcI60, are derivatives of pUC19 and maintained with at least 50 copies per cell. ${ }^{18}$ Transformants were grown to mid-log phase and the fluorescence of individual cells compared (Table 2 ).

We observed decreased fluorescence in the presence of multicopy $c I$. We interpret the reduced fluorescence of $\mathrm{KB} 126 \mathrm{pcI} I^{+}$when compared with the $c I^{-}$control, KB126pcI60, to reflect repression of $p R M$. We cannot assess the degree of repression, since the residual fluorescence produced by $\mathrm{KB}^{2} 26 \mathrm{pl}^{+}$approaches our threshold of detection, the auto-fluorescence observed with the KB124 which lacks $g f p$. Repression of $p R M$ by multicopy expression of repressor was reported earlier. ${ }^{5}$ Therefore the rex::gfp fusion showed proper regulation.

We also examined the response of a lysogen lacking oL, SS122 (Table 2). Its fluorescence was also strongly repressed by $\mathrm{pcI}^{+}$indicating proper regulation of $p R M$. The unrepressed fluorescence observed with SS122pcI60 is substantially higher than that of the $o L^{+}$strain, KB126pcI60. Increased expression from $p R M$ in the absence of $o L$ has been described (Figure 2). ${ }^{10}$

Intriguing results were obtained with pSS5. Like $\mathrm{pcI}^{+}$, pSS5 has the lac promoter placed upstream from $c I$. However, pSS5, unlike $\mathrm{pcI}^{+}$, retains $o R$. In the majority of our experiments a fraction of the cells harboring pSS5 fluoresced as strongly as the pUC18 control (Table 2). We suggest the strongly fluorescing cells result from an interaction between $o R$ retained on pSS5 and the operators of the prophage. In support of this conclusion we note

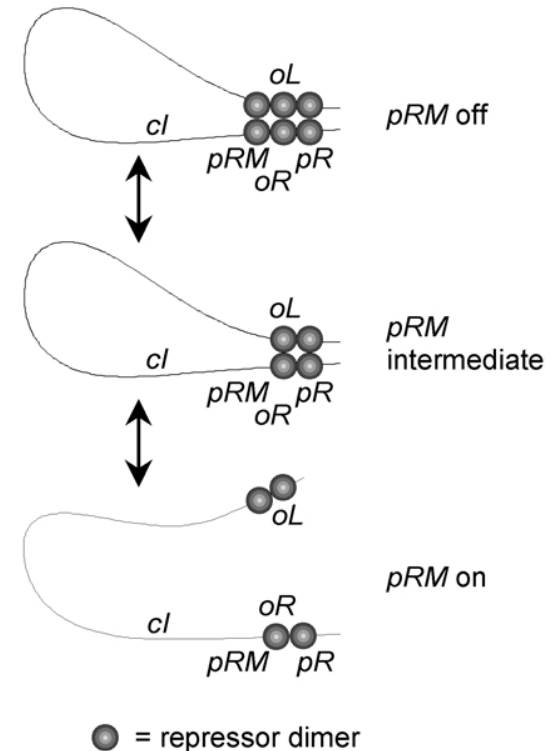

Figure 2. Model of $o L-C I-o R$ complex. In this model, ${ }^{9-11} p R M$ can be open, $p R M$ can be partially repressed in a higher-order complex formed by repressor as an octamer ${ }^{10}$ and $p R M$ can be more strongly repressed by an additional tetramer of repressor binding to $o L 3$ and $o R 3$.

that when KB126 or SS122 was made $l a c I^{\mathrm{Q}}$ by transduction, the fluorescence of the weakly fluorescing cells decreased with added IPTG but the fluorescence of the strongly fluorescing cells was insensitive to IPTG (data not shown). Thus, the bimodal distribution observed with pSS5 is not due to repressor becoming insoluble in a fraction of the cells due to a high concentration. We suggest $o R$ of pSS5 formed an aberrant complex with repressor and $o R$ of the prophage and interfered with the ability of excess repressor to inhibit $p R M$. Lastly, we observed a bimodal distribution of $g f p$ expression in the absence of recombination (the recA strain, SS74pSS5; data not shown) indicating recombination is not necessary to form the de-repressed population.

\section{Distribution of fluorescence in single cells}

We measured the fluorescence of individual cells with the goal of inferring features of $c I$ regulation from the cell-to-cell distribution of fluorescence. A micrograph of one strain examined, SS122, is shown in Figure 3. In Figure 4 we show the distribution of intensity for KB126 (Materials and Methods).

To better understand the distribution we compared it with a computer simulation of the production of GFP molecules (Materials and Methods). Since $g f p$ is preceded by an efficient site for translation initiation ${ }^{15}$ and $c I$ by an inefficient site for translation initiation, ${ }^{19}$ we assume there is at least as much GFP as repressor produced from each transcript initiated at $p R M$. In terms of our model, we make the conservative assumption that 


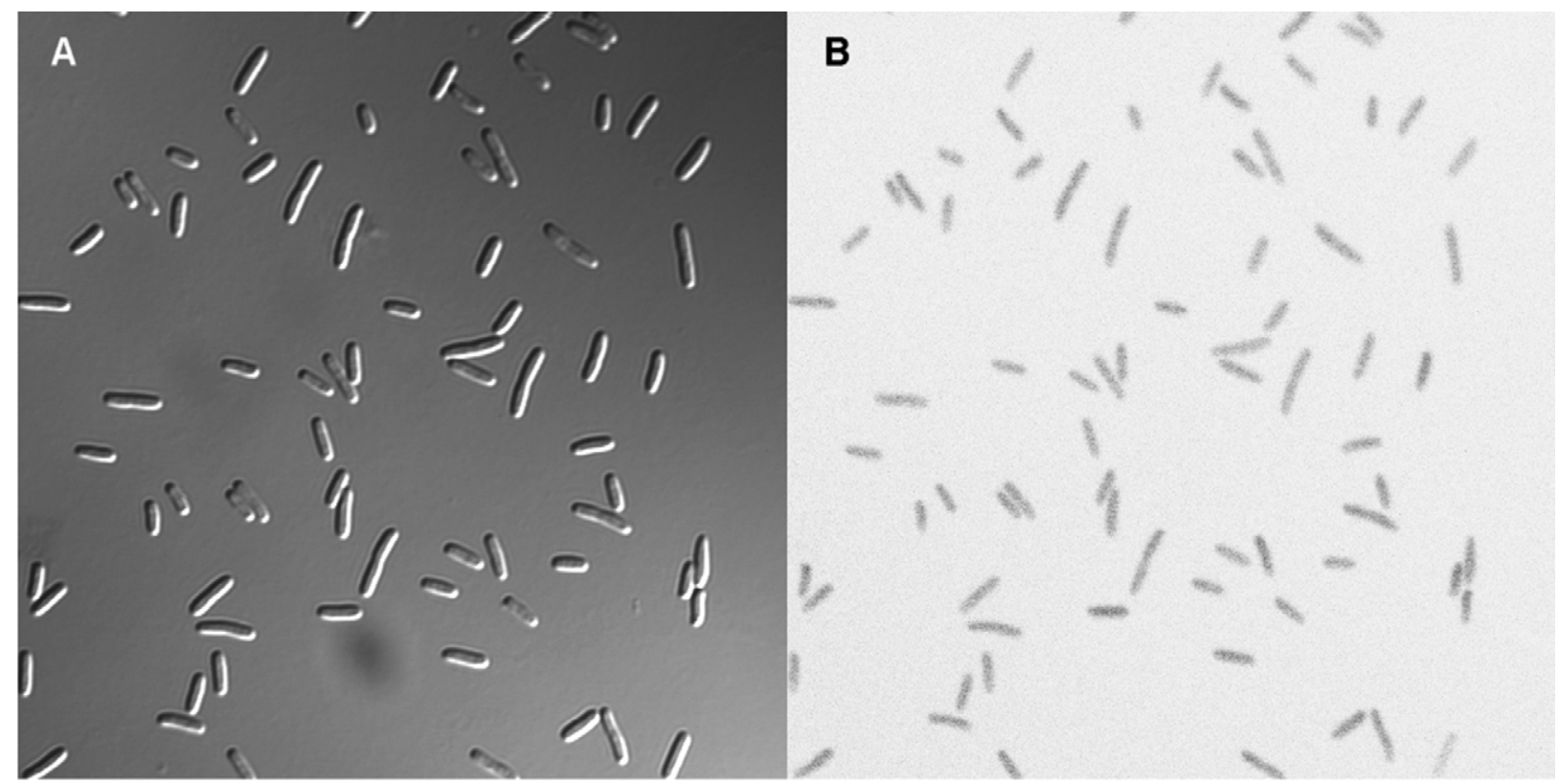

Figure 3. Confocal microscopy images. The strain shown is SS122. A, The differential interference contrast image; B, the negative image of the fluorescence.

one GFP molecule is produced per repressor molecule. Furthermore, our model assumes that production of GFP and repressor is a random stochastic process, and that dilution of both GFP and repressor only takes place upon cell division due to their observed stability. ${ }^{15,20}$ That is, at cell division each molecule in the cell randomly segregates between the two daughter cells. The predicted distribution of GFP concentrations is also shown in Figure 4.

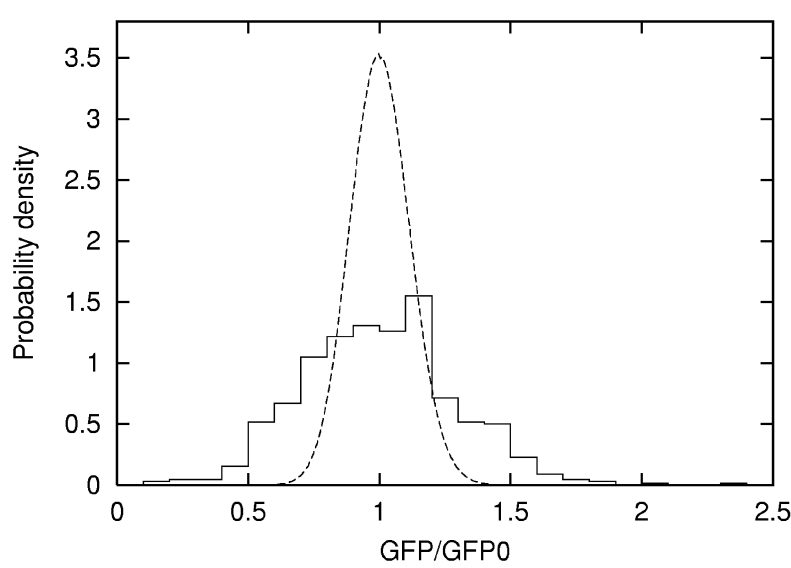

Figure 4. Distribution of GFP. The Figure shows the measured fluorescence of KB126 (continuous line) after growth in YT broth and the results of a simulation of GFP production (broken line). The fluorescence of KB126 was determined on 659 cells. The $X$ axis shows measured or predicted fluorescence intensity of individual cells divided by the average fluorescence intensity of all cells measured in the field. The $Y$ axis shows the probability density or the probability per unit interval. The simulation (Materials and Methods) assumes 70 transcription initiations from $p R M$ randomly distributed through the cell cycle.
It can be seen that the model predicts a distribution of GFP that is much narrower than observed. If $\mathrm{KB126}$, a $\mathrm{rec}^{+}$strain, was partially induced it would display a broader distribution than an isogenic $\operatorname{rec} A$ strain. We examined the distribution of GFP in the isogenic recA strain, SS74, and found the relative distribution of fluorescence intensities was very similar in the two strains, but slightly broader in the $\mathrm{rec}^{+}$strain $($ KB126 $\sigma /$ mean $=0.29 ;$ SS74 $\sigma /$ mean $=0.26)$. Although if no feature of a $\mathrm{rec}^{+}$strain broadens the distribution, gene expression in $r e c A$ strains is expected to vary more than in the isogenic $\mathrm{rec}^{+}$ strain, ${ }^{21}$ we believe the differences observed between the $\mathrm{rec} A$ and $\mathrm{rec}^{+}$strains are minor. We suggest the resistance to perturbations caused by altering the $\operatorname{rec} A$ gene, as with the tolerance of symmetric operators, ${ }^{12}$ is due to the regulatory capacity of the $c I$ expression system.

To test whether the observed distribution was an artifact of the microscopy we only analyzed bacteria that were precisely in the focal plane of the image and conducted the experiment with different pinhole diameters. Since depth of field varies with pinhole diameter, a variation due to imaging of the cells would show a dependence on pinhole diameter. We observed similar distributions with different pinhole diameters indicating the distribution was unlikely to be an artifact of the microscopy. Another potential source of broad fluorescence distribution could be fluctuations in background autofluorescence. We measured fluorescence in strains isogenic to SS74 and KB126 but lacking $g f p$, S2463 and KB124, and found autofluorescence could not account for the observed breadth of the distribution. Therefore, the observed breadth of the distribution was a property of the lysogens. 
In our simulation, the stability of the prophage would not be substantially reduced by the fluctuations in repressor concentrations per se, such as observed for GFP concentrations. This is because failure of $c I$ expression would have to occur for several generations in order to deplete the intracellular pool of repressor. Thus, the observed fluctuations of GFP concentrations are compatible with the observed stability of the prophage. However, if the observed fluctuations were due to an effect of Cro, an antagonist of repressor synthesis, then in the absence of additional mechanisms to control $c I$ expression, the predicted instability is incompatible with the observed stability of the prophage.

\section{UV experiment}

To search for a mechanism to maintain $c I$ expression in the presence of the large fluctuations, we examined the response of the rex::gfp expression to reductions in repressor concentrations such as those induced by DNA damage. We expected increased repressor synthesis in response to DNA damage could compensate for the cell-to-cell variation and permit the prophage to regulate departure from lysogeny. To investigate this possibility, we examined the response of the cI operon expression to UV irradiation in the $\mathrm{Rec}^{+}$ lysogen.

Cultures of KB126 were exposed to various doses of UV irradiation and both GFP production and phage induction were measured (Materials and Methods). The results of this experiment are shown in Figure 5. It can be seen that fluorescence increased with doses of UV that induced only a small fraction of the lysogens to enter lytic development. The greatest increase in fluorescence was approximately twice the uninduced level. With higher UV doses, more lysogens were induced but further increase in fluorescence was not observed. De-repression of $c I$ expression at very low doses of UV coincides with the depression of the inital portion of the phage release versus UV dose curve (summarized in Figure 5C). This break in the phage release curve was observed with both the rex:: gfp lysogen, KB126, and the rex lysogen, KB124 (Figure 5A) although the rex:: $g f p$ lysogen was less sensitive to UV. An interaction between Rex and repressor was suggested earlier. ${ }^{22}$ The coincidence of the break in the phage release curve and the plateau in $g f p$ expression suggests the likelihood phage will depart lysogeny in response to small amounts of DNA damage is reduced by de-repression of $c I$.

To investigate the contribution of the $o L-C I-O R$ complex $^{9,10}$ (Figure 2) to the UV-mediated de-repression of $p R M$, we deleted $o L$ from the prophage of KB126. If the prophage contained a large deletion that included oL (SS122; Figure 5B) the induction of GFP synthesis by UV was not observed. We suggest the differences in GFP induction between KB126 and SS122 was due to
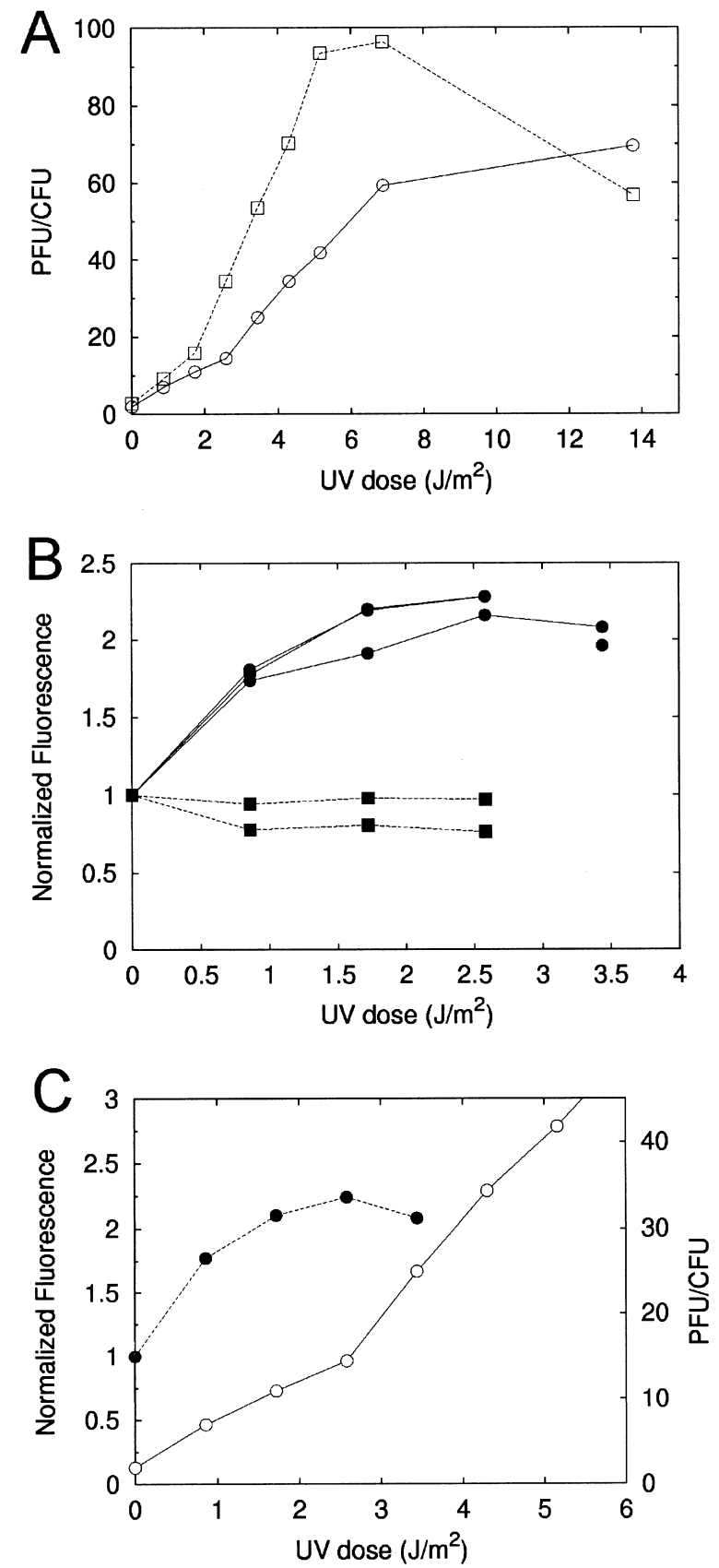

Figure 5. Response to low doses of UV. KB124, KB126 and SS122 were irradiated with the indicated dose of UV. Phage release per irradiated cell and fluorescence was measured (Materials and Methods). A, Phage release per irradiated cell of KB124 (open squares) and KB126 (open circles). Averages of three experiments are shown. B, The fluorescence of KB126 (filled circles) and SS122 (filled squares). Normalized fluorescence is the average fluorescence intensity of irradiated cells divided by the average fluorescence intensity of non-irradiated cells from the same culture. Each curve is derived from an independent culture. Un-irradiated SS122 cells had approximately three times the fluorescence of unirradiated KB126 cells. Fluorescence intensity of KB124 (auto-fluorescence) was too low for quantification and did not increase in response to UV. C, Summary of the results shown in A and B for KB126: phage release (open circles) and fluorescence (filled circles). Fluorescence values are the average of the values reported in B. 
the presence of $o L$ and the induction in KB126 was due to opening of the $o L-\mathrm{CI}-o \mathrm{R}$ complex. The average fluorescence of SS122 in this experiment was 31 units while the fluorescence of KB126 following UV exposure peaked at 23 units. Thus, in spite of induction by UV, the prophage retaining $o L$ did not attain the high level of expression observed for the prophage lacking $o L$.

$\lambda$ repressor can exist as a monomer, a dimer and a dimer bound to the $\lambda$ operators. Exchange between these states is rapid compared with the generation time of E. coli $\left(\right.$ review $\left.^{3}\right)$. The form of repressor, which degrades via activated RecA in response to DNA damage, is the monomer form $\left(\right.$ review $\left.^{2}\right)$. We suggest the depletion of repressor monomers results in opening the $o L-C I-O R$ complex increasing transcription of $c I$ from $p R M$ (Figure 2). The increased expression of the $c I$ operon in response to low exposure to a DNAdamaging agent prevents premature departure from the lysogenic state. This increase in $\mathrm{cI}$ operon expression helps the prophage tolerate fluctuations in repressor concentrations while maintaining lysogeny. However, great increases in $c I$ expression would prevent the prophage from departing lysogeny after severe DNA damage. The limited increase of GFP content observed in KB126 permits the prophage to efficiently depart lysogeny after higher doses of UV.

\section{Conclusion}

The $\lambda$ prophage is remarkably stable. In $\operatorname{rec} A$ strains, the majority of phage that have departed lysogeny are mutated in the repressor, i.e. they form clear plaques. Furthermore, turbid plaques, those previously assumed to be wild-type and result from failure of the lysogenic regulatory network, are also the result of mutation. The turbid plaques released from recA strains are defective in the maintenance of repression. Thus, the $\lambda$ prophage is vastly more stable than previously assumed. Furthermore, the stability prevails in the absence of the second known promoter for $c I$ transcription, $p R E$.

The profound stability of the lysogenic state is not due to exceptionally precise control of repressor concentrations. In fact, the concentration of repressor varies widely from cell-to-cell. The fluctuation in repressor concentrations does not lead to instability of the prophage, since the prophage regulatory network can compensate for substantial decreases in repressor concentrations. The response to low doses of UV presumably occurs through this compensatory response, since the prophage remains profoundly stable in recA strains, which cannot mount an SOS-response. The response to low doses of UV required the presence of $o L$ and we suggest the response occurs through the $o L-C I-o R$ complex. In spite of a UV-induced increase in repressor synthesis, the prophage can effectively respond to greater DNA damage and depart lysogeny. The network retains its ability to respond to DNA damage because of the limited induction ratio for repressor synthesis.

\section{Materials and Methods}

\section{Plasmids}

The immunity region (base-pairs 34,500 to 39,173 ) was cloned from $\lambda^{+}$as the BamHI-EcoRI fragment replacing the BclI-EcoRI fragment of pACYC184 ${ }^{23}$ to create pSS1. The PstI-PflFI fragment of rexA-rexB (base-pairs $37,006-36,124)$ was replaced with the XbaI-HindIII fragment containing $g f p$ from pGFPmut $2^{15}$ to create pSS2. The fragment ends were made blunt by T4 DNA polymerase for the portion from pSS1 and by Klenow fragment of E. coli DNA polymerase for the portion from pGFPmut2. pSS5 places $c I$ under the control of the lac promoter and contains the BglII-PstI fragment of $\lambda^{+}$ (base-pairs 38,107-37,001) replacing the BamHI-PstI fragment of pUC18. ${ }^{24}$

$\mathrm{pcI}^{+}$and pcI60 were prepared by amplifying basepairs 37,218-37,959 from either $\lambda^{+}$or $\lambda$ cI60. Oligonucleotides $5^{\prime}$ CGCGGATCCGCGGTGATAGATTTAACG TATG and 5'CCGGAATTCCCTTGCCGATCAGCCAAA CGTC and KOD Polymerase (Novagen) was used to amplify base-pairs $37,218-37,959$, the product digested with BamHI and EcoRI replacing the BamHI-EcoRI fragment of pUC19. ${ }^{24}$

A fragment containing $508 \mathrm{bp}$ internal to the galK gene was amplified using pCNP4 ${ }^{25}$ as a template and primers $5^{\prime}$ GGTGAAACATCTGCAGCTGCGTAACAAC $3^{\prime}$ and $5^{\prime}$ TATATGACGCACGCGTTTTGCC $3^{\prime}$. The fragment was introduced into pSS2 replacing the AhdI-PstI fragment. The PstI site is located immediately downstream of the stop codon of $g f p$. A PstI fragment from pSB590 $0^{26}$ encoding kanamycin resistance was inserted at the PstI site between $g f p$ and the $g a l K$ fragment to yield pKB85.

\section{Strains and media}

All bacterial strains are derivatives of E. coli $\mathrm{K} 12$ and are described in Table 3. The parental strains were MC4100, MG1655, S971 and SY822. The rex:: gfp fusion was crossed with $\lambda i m m 434$ by transforming SS16 with pSS2 and mating with SS15. Zygotically induced phage were purified on SS10. Single lysogens were identified by the PCR assay described by Powell et al. ${ }^{27}$ using primers: $b 2$ side of $a t t P$, GTATGCATGCTGGGTGTGGG GAA; int side of attP, CGCGCACGAAAAGCATCAGGT; gal side of attP, CCGACAGAATCGGGCGAGAAGA; and bio side of attB, CTCTCTGGCAAGCGCCTCGATT. All $\lambda$ imm $\lambda$ strains, except S2245 were determined to be single lysogens. Bacteria were grown in YT medium ${ }^{28}$ unless stated. Ampicillin was added to $200 \mu \mathrm{g} / \mathrm{ml}$, chloramphenicol to $25 \mu \mathrm{g} / \mathrm{ml}$, kanamycin to $30 \mu \mathrm{g} / \mathrm{ml}$, streptomycin to $100 \mu \mathrm{g} / \mathrm{ml}$ and tetracycline to $10 \mu \mathrm{g} / \mathrm{ml}$ as required.

SS122 was constructed by recombination with pKB85 as described. ${ }^{26}$ pKB85 was transformed into S971 lysogenized with $\lambda r e x:: g f p$ and mated with KB126 selecting for streptomycin and kanamycin resistance. P1vir was grown on the resistant population and used to transduce KB126 to kanamycin resistance. SS122 was identified as a Gal ${ }^{-}$, tetracycline-sensitive transductant that did not release phage. 
Table 3. E. coli strains used in this study

\begin{tabular}{|c|c|c|}
\hline Strain & Genotype & Reference \\
\hline KB12 & $\mathrm{F}^{-}$rph srl:: Tn10 $\Delta(\operatorname{srl}-\mathrm{rec} A) 306 \operatorname{lamB}\left(\lambda^{+}\right)$ & This study \\
\hline KB13 & $\mathrm{F}^{-}$rph srl:: Tn10 $\Delta($ srl-recA)306 lamB $(\lambda c y 42)$ & This study \\
\hline KB14 & $\mathrm{F}^{-}$rph srl:: Tn10 $\Delta($ srl-recA)306 lamB $(\lambda c y 2001)$ & This study \\
\hline KB15 & $\mathrm{F}^{-}$rph srl :: Tn10 $\Delta(\operatorname{srl}-\mathrm{rec} A) 306$ lamB $(\lambda$ cII68) & This study \\
\hline KB16 & $\mathrm{F}^{-}$rph srl :: Tn10 $\Delta($ srl-recA)306 lamB $(\lambda c I I 2002)$ & This study \\
\hline KB124 & $\mathrm{F}^{-} \Delta\left(\right.$ lacl-Z)M455 his arg rpsL31 lamB $\left(\lambda^{+}\right)$ & This study \\
\hline KB126 & $\mathrm{F}^{-} \Delta($ lacI-Z)M455 his arg rpsL31 lamB $(\lambda$ rex:: $g f p)$ & This study \\
\hline MC4100 & $\mathrm{F}^{-}$slacx74 araD139 thi rpsL & 34 \\
\hline MG1655 & $\mathrm{F}^{-} r p h$ & 35 \\
\hline S971 & $\mathrm{HfrH} l a c I^{\mathrm{Q}}$ & 36 \\
\hline S2245 & $\mathrm{F}^{-}$- $l a c x 74$ araD139 thi rpsL fhuA, $(\lambda c 1857)$ & This study \\
\hline S2463 & $\mathrm{F}^{-} \Delta\left(\right.$ lacl-Z)M455 $\Delta($ srl-rec $A) 304$ his arg rpsL31 lamB $\left(\lambda^{+}\right)$ & This study \\
\hline S2480 & $\mathrm{F}^{-}$rph srl ::Tn10 $\Delta(\operatorname{srl}-r e c A) 306$ & 3 \\
\hline SS10 & $\mathrm{F}^{-}$dlacx74 araD139 thi rpsL $(\lambda i m m 434)$ & This study \\
\hline SS15 & $\mathrm{F}^{-}$- $l a c x 74$ araD139 thi rpsL lamB (גimm434) & This study \\
\hline SS16 & HfrH lacI $\operatorname{lamB}(\lambda i m m 434)$ & This study \\
\hline SS74 & $\mathrm{F}^{-} \Delta($ lacl-Z)M455 $\Delta($ srl-recA $) 304$ his arg rpsL31 lamB $(\lambda$ rex:: gfp) & This study \\
\hline SS122 & $\mathrm{F}^{-} \Delta($ lacl-Z)M455 his arg rpsL31 lamB $(\lambda$ rex $:: g f p) \Delta($ rex-galK $)::$ kan & This study \\
\hline SY822 & $\mathrm{F}^{-} \Delta($ lacl-Z)M455 $\Delta($ srl-rec $A) 304$ his arg rpsL31 & 37 \\
\hline
\end{tabular}

All strains are derivatives of E. coli $\mathrm{K} 12$.

\section{Complementation analysis}

Liquid lysates were made from individual plaques as described by Silhavy et al. ${ }^{29}$ and titers of the lysates were determined. A saturated culture of S2245 grown at $30{ }^{\circ} \mathrm{C}$ was spread at several concentrations onto $\mathrm{T}$ agar and allowed to dry. Dilutions of the lysates were spotted in $10 \mu \mathrm{l}$ drops on the lawn of bacteria and colonies were counted after overnight incubation at $42^{\circ}$. To calculate multiplicity of infection (m.o.i.) the area of the spots was measured to determine $\mathrm{PFU} / \mathrm{cm}^{2}$, which was divided by $\mathrm{CFU} / \mathrm{cm}^{2}$. Bacteria and phage were combined to generate m.o.i. values between $10^{-4}$ and $10^{-1}$. Efficiency of transduction was determined from the linear portion of the relationship of colonies formed at $42{ }^{\circ} \mathrm{C}$ to input PFU.

\section{Phage release}

Overnight cultures were washed with M63 salts and resuspended in YT supplemented with $10 \mathrm{mM} \mathrm{MgSO}_{4}$. The washed cultures were diluted 1000 -fold into $20 \mathrm{ml}$ of YT supplemented with $10 \mathrm{mM} \mathrm{MgSO}_{4}$ and grown to $5 \times 10^{7} \mathrm{CFU} / \mathrm{ml}$. Aliquots were removed and viable cells quantified by colony formation. The remainder of each culture was sterilized with $\mathrm{CHCl}_{3}$ and filtered through a $0.2 \mu \mathrm{m}$ pore size filter. The filtrates were titered on MC4100 to quantify phage released.

\section{Microscopy and data collection}

Cultures in mid-log phase were mixed 1:1 with $100 \mu \mathrm{g} / \mathrm{ml}$ of spectinomycin in $10 \mathrm{mM}$ sodium phosphate, $0.1 \mathrm{M} \mathrm{NaCl}(\mathrm{pH} 7)$. Samples $(14 \mu \mathrm{l})$ were transferred to washed, agarose-coated slides. ${ }^{30}$ Samples were examined as wet-mounts by laser scanning confocal microscopy. Fluorescence was quantified by measuring the average gray scale value in the fluorescent channel along the midline extending the length of the cell. When only the distribution of fluorescence among cells was examined, the data from different images were pooled by setting the average fluorescence intensity of cells in each image to 1.

\section{UV irradiation}

Cells were grown to mid-log phase and washed in pre-warmed $0.1 \mathrm{M} \mathrm{MgSO}_{4}$. Washed cells were irradiated with a $254 \mathrm{~nm} \mathrm{UV}$ source at $0.09 \mathrm{~J} / \mathrm{m}^{2}$ per second in dim ambient light. After UV irradiation cell suspensions were diluted 1:1 in pre-warmed YT and shaken in the dark. After 30-40 minutes an aliquot was removed for microscopy and treated as described. Incubation was continued for four hours at $37^{\circ} \mathrm{C}$. The cultures were sterilized with $\mathrm{CHCl}_{3}$, cell debris removed by centrifugation and the released phage titered. The concentration of viable cells was measured immediately before UV treatment.

\section{Fluctuation analysis and computer simulation}

To translate the observed level of fluctuations into cellular mechanisms we employed an extended version of the stochastic model described by Aurell et al., taking into account both $o L-o R$ interactions for negative autoregulation of $p R M$ developed to fit the $p R M$ activity data by Dodd et al.,10 and supplemented by a special treatment for GFP production and segregation into daughter cells. In practice this involves the inclusion of 27 different patterns of $\mathrm{CI}$ and Cro bindings to $o L$ bindings, 40 different bindings of RNA polymerase, CI and Cro to $o R$, and inclusion of $o L-C I-O R$ binding of, respectively, $-1 \mathrm{kcal} / \mathrm{mol}(1 \mathrm{cal}=4.184 \mathrm{~J})$ for octamer association, and a $-3 \mathrm{kcal} / \mathrm{mol}$ for a full 12 -mer binding of $C I$ on all $o L$ sites to $C I$ at all $o R$ operator sites. Finally, from the $p R M-C I$ data by Dodd et al. ${ }^{10}$ and the $p R-C$ ro data by Pakula et al. ${ }^{31}$ as discussed by Reinitz \& Vaisnys ${ }^{32}$ we estimate $\mathrm{CI}$ and Cro non-specific binding to both be $-3.5 \mathrm{kcal} / \mathrm{mol}$. GFP is subsequently introduced as a passive ingredient in the model, and is always produced in conjunction with $\mathrm{CI}$ but is not negatively self-regulated. Thus, the fluctuations of GFP are slightly larger than for CI levels.

The basic ingredient in a stochastic model for protein production is a production term given by a number of mRNA transcripts and a fluctuating number of proteins per transcript (see Aurell et al. ${ }^{3}$ ). In addition, when the 
cells divide all molecules should be randomly distributed to the two daughter cells. The resulting fluctuations in GFP concentration will be of order $N_{\text {event }}^{-1 / 2}$ where $N_{\text {event }}$ is the number of independent events that control the amount of GFP in the cell. However, because each cell division limits the overall meandering of concentration of any protein in a cell, a more precise estimate of fluctuations needs a simulation. However, in practice we find that the spread over mean for the GFP signal is well fitted by:

$$
\frac{\sigma}{\text { mean }}=\frac{1}{\sqrt{N_{\mathrm{mRNA}}}}
$$

where $N_{\text {mRNA }}$ is the number of CI-GFP mRNA transcript produced in a cell generation. The simulation assumed 70 transcripts per cell cycle were initiated at $p R M$ to generate 170 repressor monomers. This efficiency of translation of $C I$ is derived from the ratio of translation of $c I$ from $p R M$ to lacZ from its own ribosome-binding site $^{19}$ and the efficiency of translation of lacZ. ${ }^{33}$ The number of repressor monomers per cell is derived from the concentration of repressor in lysogens ${ }^{14}$ and the molecular mass of the $\lambda$-repressor.

\section{Acknowledgements}

We thank Sankar Adhya, Kasper Eriksen, Max Gottesman, Anders Løbner-Olesen, Michael Sørensen and Genevieve Thon for helpful suggestions. We thank the Novo Nordisk Foundation for support.

\section{References}

1. Ptashne, M. (1992). A Genetic Switch, Cell Press, Blackwell Scientific Publications, Cambridge, MA.

2. Roberts, J. W. \& Devoret, R. (1983). Lysogenic induction. In Lambda II (Hendrix, R. W., Roberts, J. W., Stahl, F. W. \& Weisberg, R. A., eds), pp. 123-144, Cold Spring Harbour Laboratory Press, Cold Spring Harbor, NY.

3. Aurell, E., Brown, S., Johanson, J. \& Sneppen, K. (2002). Stability puzzles in phage. Phys. Rev. ser. E, 65, 05191.

4. Brooks, K. \& Clark, A. J. (1967). Behavior of $\lambda$ bacteriophage in a recombination deficient strain of Escherichia coli. J. Virol. 1, 283-293.

5. Maurer, R., Meyer, B. \& Ptashne, M. (1980). Gene regulation at the right operator (OR) bacteriophage $\lambda$. I. OR3 and autogenous negative control by repressor. J. Mol. Biol. 139, 147-161.

6. Meyer, B. J., Maurer, R. \& Ptashne, M. (1980). Gene regulation at the right operator (OR) of bacteriophage $\lambda$. II. OR1, OR2, and OR3: their roles in mediating the effects of repressor and cro. J. Mol. Biol. 139, 163-194.

7. Meyer, B. J. \& Ptashne, M. (1980). Gene regulation at the right operator (OR) of bacteriophage $\lambda$. III. $\lambda$ repressor directly activated gene transcription. J. Mol. Biol. 136, 195-205.

8. Johnson, A. D., Poteete, A. R., Lauer, G., Sauer, R. T., Ackers, G. K. \& Ptashne, M. (1981). $\lambda$ repressor and cro-components of an efficient molecular switch. Nature, 294, 217-223.
9. Révet, B., von Wilcken-Bergmann, B., Bessert, H., Barker, A. \& Muller-Hill, B. (1999). Four dimers of lambda repressor bound to two suitably spaced pairs of lambda operators form octamers and DNA loops over large distances. Curr. Biol. 9, 151-154.

10. Dodd, I. B., Perkins, A. J., Tsemitsidis, D. \& Egan, B. J. (2001). Octamerization of $\lambda$ CI repressor is needed for effective repression of $\mathrm{pRM}$ and efficient switching from lysogeny. Genes Dev. 15, 3013-3022.

11. Bell, C. E., Frescura, P., Hochschild, A. \& Lewis, M. (2000). Crystal structure of the $\lambda$ repressor C-terminal domain provides a model for cooperative operator binding. Cell, 101, 801-811.

12. Little, J. W., Shepley, D. P. \& Wert, D. W. (1999). Robustness of a gene regulatory ciruit. $E M B O \mathrm{~J} . \mathbf{1 8}$, 4207-4299.

13. Yen, K.-M. \& Gussin, G. N. (1973). Genetic characterization of a $\mathrm{prm}^{-}$mutant of bacteriophage $\lambda$. Virology, 56, 300-312.

14. Reichardt, L. \& Kaiser, A. D. (1971). Control of $\lambda$ repressor synthesis. Proc. Natl Acad. Sci. USA, 68, 2185-2189.

15. Cormack, B. P., Valdivia, R. H. \& Falkow, S. (1996). FACS-optimized mutants of the green fluorescent protein (GFP). Gene, 173, 33-38.

16. Løbner-Olesen, A. (1999). Distribution of minichromosomes in individual Escherichia coli cells: implications for replication control. $E M B O \mathrm{~J} . \mathbf{1 8}$, 1712-1721.

17. Kalir, S., McClure, J., Pabbaraju, K., Southward, C., Ronen, M., Leibler, S. et al. (2001). Ordering genes in a flagella pathway by analysis of expression kinetics from living bacteria. Science, 292, 2080-2083.

18. Lutz, R. \& Bujard, H. (1997). Independent tight regulation of transcriptional units in Escherichia coli via the LacR/O, the TetR/O and AraC/I1-I2 regulatory elements. Nucl. Acids Res. 25, 1203-1210.

19. Shean, C. S. \& Gottesman, M. E. (1992). Translation of the prophage $\lambda c I$ transcript. Cell, 70, 513-522.

20. Reichardt, L. F. (1975). Control of bacteriophage lambda repressor synthesis: regulation of the maintenance pathway by the cro and $c I$ products. J. Mol. Biol. 93, 289-309.

21. Elowitz, M. B., Levine, A. J., Siggia, E. D. \& Swain, P. S. (2002). Stochastic gene expression in a single cell. Science, 297, 1183-1186.

22. Eisen, H., Pereira da Silva, L. H. \& Jacob, F. (1968). Sur la regulation precoce du bacteriophage. C.R. Acad. Sci. Paris, 266, 1176-1178.

23. Chang, A. C. Y. \& Cohen, S. N. (1978). Construction characterization of amplifiable multicopy DNA cloning vehicles derived from the P15A cryptic miniplasmid. J. Bacteriol. 134, 1141-1156.

24. Yanisch-Perron, C., Vieira, J. \& Messing, J. (1985). Improved M13 phage cloning vectors and host strains: nucleotide sequences of the M13mp18 and pUC19 vectors. Gene, 33, 103-119.

25. Petersen, C. (1987). The functional stability of the lacZ transcript is sensitive towards sequence alterations immediately downstream of the ribosome binding site. Mol. Gen. Genet. 209, 179-187.

26. Brown, S. \& Fournier, M. J. (1984). The 4.5 S RNA gene of E. coli is essential for cell growth. J. Mol. Biol. 178, 533-550.

27. Powell, B. S., Rivas, M. P., Court, D. L., Nakamura, Y. \& Turnbough, C. L. (1994). Rapid confirmation of single copy lambda prophage integration by PCR. Nucl. Acids Res. 22, 5765-5766. 
28. Miller, J. H. (1972). Experiments in Molecular Genetics, Cold Spring Harbor Laboratory Press, Cold Spring Harbor, NY.

29. Silhavy, T. J., Berman, M. L. \& Enquist, L. W. (1984) Experiments with Gene Fusions, Cold Spring Harbor Laboratory Press, Cold Spring Harbor, NY.

30. Lemon, K. P. \& Grossman, A. D. (1998). Localization of bacterial DNA polymerase: evidence for a factory model of replication. Science, 282, 1516-1519.

31. Pakula, A., Young, V. \& Sauer, R. (1986). Bacteriophage $\lambda$ cro mutations: effect on activity and intracellular degradation. Proc. Natl Acad. Sci. USA, 83, $8829-8833$.

32. Reinitz, J. \& Vaisnys, J. R. (1990). Theoretical and experimental analysis of the phage $\lambda$ genetic switch impies missing levels of co-operativity. J. Theor. Biol. 145, 295-318.

33. Kennell, D. \& Riezman, H. (1977). Transcription translation initiation frequencies of the Escherichia coli lac operon. J. Mol. Biol. 114, 1-21.

34. Casadaban, M. J. (1976). Transposition fusion of the lac genes to selected promoters in E. coli using bacteriophage lambda and mu. J. Mol. Biol. 104, 541-555.

35. Jensen, K. F. (1993). The Escherichia coli K-12 "wild types" W3110 and MG1655 have an rph frameshift mutation that leads to pyrimidine starvation due to low pyrE expression levels. J. Bacteriol. 175, 3401-3407.

36. Brown, S. (1987). Mutations in the gene for EF-G reduce the requirement for $4.5 \mathrm{~S}$ RNA in the growth of E. coli. Cell, 49, 825-833.

37. Isberg, R. R., Lazaar, A. L. \& Syvanen, M. (1982). Regulation of Tn 5 by the right-repeat proteins: control at the level of the transposition reaction? Cell, 30, 883-892.

Edited by M. Gottesman

(Received 23 June 2003; received in revised form 10 September 2003; accepted 15 September 2003) 